Remise de cabinets médicaux

\& recherche d'associés

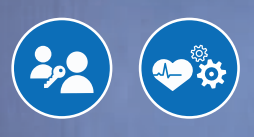

En tant que fournisseur de prestations propre au corps médical, nous offrons:

- Une grande expérience, des compétences approfondies et une large reconnaissance

- Un vaste réseau en Suisse et à l'étranger

- Une neutralité absolue, car vendeurs et acheteurs sont nos sociétaires

- Un soutien et un accompagnement global

- Un excellent rapport qualité-prix

\title{
REMISE DE CABINETS MÉDICAUX ET RECHERCHE D’ASSOCIÉS
}

\section{DFMHSERVICES}

Vous pouvez prendre contact avec nous sans aucune obligation par téléphone ou courrier électronique ou en nous retournant le talon-réponse par fax ou par la Poste. Nous vous contacterons.

Prénom / nom

Adresse

NPA / lieu

Téléphone privé / prof.

Atteignable de préférence vers

Adresse mail

\section{CONSULTING}

\section{FMH Consulting Services AG}

Avenue d'Ouchy 66, 1006 Lausanne

Tél. 0219224435

mail@fmhconsulting.ch - www.fmhservices.ch 